\title{
Role of free radicals and certain antioxidants in the management of huntington's disease: a review
}

\begin{abstract}
Antioxidants are the agents believed to quench the free radicals generation from an individual who start the stages of neurodegeneration. Free radicals are atoms that contain one or more unpaired electrons because of this property which makes them highly reactive. Because of this property these free radicals attached very quickly on the defective cell or that area causes a micro crack in the mitochondrial cell, causes cell damage. Oxidative free radicals are generated by metabolic reactions which can be creating a chain reaction with leading to membrane lipid per-oxidation and DNA damage at the cellular level in the neurons. Free radical has been implicated in various neurodegenerative diseases such as Huntington \& Alzheimer's disease. Many endogenous and dietary compounds like vitamin $\mathrm{E}$ and $\mathrm{C}$, superoxide dismutase, coenzyme Q10, ferritin, transferring, ceruloplasmin, $\alpha$ tocopherol, $\beta$ carotene, $\alpha$ lipoate, lycopene and ascorbic acid have antioxidant and free radical scavenging properties that help to quench free radical in HD and delay the oxidative damage. Antioxidant can be used to defense the mitochondrial damage and several mechanisms include removal of $\mathrm{O} 2$, scavenging of reactive oxygen and nitrogen species or their precursors, inhibition of ROS formation and binding of metal ions needed for the catalysis of ROS, generation and up-regulation of endogenous antioxidant defenses the tissue damage which can be beneficial in HD. The present review deals with the effect of antioxidants on Huntington disease and certain antioxidants can be prepared by plant dietary fibers, fruits and some type of vegetables. Plants and polyphenols have the great role in the management of the symptoms of Huntington disease.
\end{abstract}

Keywords: huntington disease, antioxidants, reactive oxygen species, mitochondrial damage
Volume 7 Issue 4 - 2018

\author{
Firoz Khan,Vipin Kumar Garg,Avnesh Kumar \\ Singh, Tinku Kumar \\ Department of Pharmaceutical Technology, Meerut Institute of \\ Engineering and Technology, India
}

Correspondence: Firoz Khan, Department of Pharmaceutica Technology, Meerut Institute of Engineering and Technology, Meerut, N.H-58, Delhi-Roorkee Highway, Baghpat Bypass Road Crossing Meerut, U.P- 250005, India,

Email fkpharmacy@gmail.com

Received: May 14, 2018 | Published: July 04, 2018

\section{Introduction}

Huntington's disease is an autosomal dominant neurodegenerative disease caused by a mutation in the gene encoding a protein called huntingtin (htt). The mutation consists in an excess of repeats in the CAG triplet within the coding region of the IT15 gene encoding htt, resulting in a polyQ tract near the N-terminus of this protein. ${ }^{1}$ The disease is characterized by the occurrence of two major symptoms, i.e. motor abnormalities and cognitive impairment. ${ }^{2}$ Neurodegenerative process is a progressive disease but, in terms of motor symptoms, has a biphasic profile. Thus, the early stages of the disease are characterized by involuntary movements termed "chorea" that correspond to a primary affectation of striatal GABA neurons that project to the globus pallidus..$^{3-5}$ Cortical glutamatergic neurons that project to the striatum also degenerate in these early stages and this could explain the cognitive impairments and psychological disturbances also appearing in HD patients. ${ }^{6}$ Once the disease has advanced and the neuronal death is massive and also affects other striatal subpopulations, patients show a parkinsonian like symptomatology with bradykinesia and rigidity episodes. ${ }^{7}$ Although the primary cause of neurodegenerative process occurring in HD is the toxicity of the mutant htt, several additional processes, most of them common to other neurodegenerative disorders, i.e. protein misfolding, abnormal proteolysis, protein aggregation and deposition, transcriptional dysregulation, mitochondrial dysfunction, excitotoxic and oxidative events, and glial activation and local inflammatory events, have been also involved in neuronal death in HD. ${ }^{8}$ Unfortunately, HD has still no cure and patients die approximately 10-20 years after diagnosed. They only have relief therapy to alleviate some symptomatic features associated to the disease (i.e. antidopaminergic drugs to alleviate the hyperkinesia observed in the first stages. ${ }^{9}$ There was also some attempts with antiglutamatergic agents to reduce excitotoxicity effect in the damaged cell of brain but the efficacy was very limited. ${ }^{10}$ In the last years, various groups of novel compounds, i.e. unsaturated fatty acids, minocycline, coenzyme Q10, inhibitors of histone deacetylases, have been studied in preclinical models and, even, some of them have entried in the clinical evaluation phase as potential novel diseasemodifying agents in HD. ${ }^{11,12}$ Promising expectatives have been also obtained from cannabinoid compounds in cellular and animal models given their well-known neuroprotective effects (see above), even, some specific cannabinoid-related compounds have been proposed for attenuating hyperkinetic involuntary movements in HD although this possibility will not be addressed in this review article, which concentrates in their disease-modifying effects. ${ }^{13,14}$

\section{Epidemiology}

Great geographic differences were seen in HD prevalence. The overall prevalence of $\mathrm{HD}$ in Asian was 0.40/100,000 (95\% confidence interval [CI]: $0.26-0.61)$, much lower comparing with that of 5.70/100,000 (95\% CI: 4.42-7.35) in European, North American, and Australia. Recently, an epidemiologic study of HD in Taiwan (China) showed that the average annual incidence rate was $0.1 / 100,000$, much lower than those of Caucasians $(5-10 / 100,000)$. Many studies have showed that HD prevalence is closely related to the different genotypes of population. The available information on the world distribution of Huntington's disease (HD) from population surveys and death rate analysis is summarized and discussed in the light of 
genetic studies. It is concluded that most European populations, both Northern and Southern, show a relatively high prevalence (4-8 per 100,000 ), and that the disorder may also be frequent in India and parts of central Asia. HD is notably rare in Finland and in Japan, but data for Eastern Asia and Africa are inadequate. The disorder may have been underestimated in the American black population. Populations derived from recent European immigration show frequencies and origins of HD comparable to those expected from their own origins and expansion; there is no evidence to suggest that the HD gene has spread disproportionally and its selective effect may be close to neutral. Multiple separate introductions of the gene have been the rule in large populations. Several major foci of HD exist as the result of rapid population expansion. It is likely that a number of separate mutations for HD will be shown to be responsible for the disease, but that the high frequency of HD in European populations will prove to be the result of one or a very small number of mutations, probably of great antiquity. ${ }^{15}$

\section{Free radicals}

Free Radicals are molecules with an unpaired electron. Due to the presence of an unpaired free electron, these molecules are highly reactive. They are important intermediates in natural processes involved in cytotoxicity, control of vascular tone, and neurotransmission. Radiolysis is a powerful method to generate specific free radicals and measure their reactivity.

\section{Types of long lived radicals}

Stable radicals: The prime example of a stable radical is molecular oxygen $\mathrm{O}_{2}$. Organic radicals can be long lived if they occur in a conjugated $\pi$ system, such as the radical derived from $\alpha$-tocopherol \& vitamin E. Thiazyl radicals show remarkable kinetic and thermodynamic stability, with only a very limited extent of $\pi$ resonance stabilization. Persistent radicals: Compounds with persistent radicals are long lived due to steric crowding around the radical center and makes them physically difficult to react with another molecule. Examples of these include-Gomberg's triphenylmethyl radical, Fremy's salt (Potassium nitrosodisulfonate, Nitroxides, such as TEMPO (2,2,6,6-Tetramethylpiperidine-1-oxyl), verdazyls, nitronyl nitroxides, azephenylenyls, radicals derived from PTM (perchlorophenylmethyl radical) and TM tris(2,4,6trichlorophenylmethyl radical). The longest-lived free radical is melanin, which may persist for millions of years. Diradicals: Molecules containing two radical centers are called diradical. Multiple radical centers can also exist in a molecule. Molecular oxygen naturally (i.e. atmospheric oxygen) exists as a diradical (in its ground state as triplet oxygen). The high reactivity of atmospheric oxygen is owed somewhat to its diradical state (although non-radical states of oxygen are actually less stable). The existence of atmospheric molecular oxygen as a triplet-state radical is the cause of its paramagnetic character, which can be easily demonstrated by attraction of oxygen to an external magnet. ${ }^{16}$ Production route of free radicals Production of free radicals in the body is continuous and inescapable. The basic causes include the following.

\section{Formation of free radicals}

Normally, bonds do not split to leave a molecule with an odd and an unpaired electron. But when weak bonds split, free radicals are formed. Free radicals are very unstable and react quickly with other compounds, trying to capture the needed electron to gain stability. When the "attacked" molecule loses its electron, it becomes a free radical itself, beginning a chain reaction. All this happens in nanoseconds. Once the process is started, it can cascade, finally resulting in the disruption of a living cell. Some free radicals may arise normally during metabolism and by immune system's cells purposefully to neutralize viruses and bacteria. Normally, the body can handle free radicals, but if antioxidants are unavailable, or if the free radical production becomes excessive, damage can occur. ${ }^{17}$

\section{Steps involving in free radical generation}

In chemistry, free radicals take part in radical addition and radical substitution as reactive intermediates. Chain reactions involving free radicals can usually be divided into three distinct processes:
a. Initiation,
b. Propagation,
c. Termination.

Initiation reactions are those, which result in a net increase in the number of free radicals. They may involve the formation of free radicals from stable species or they may involve reactions of free radicals with stable species to form more free radicals.

Propagation reactions involve free radicals in which the total number of free radicals remains the same. Termination reactions are those reactions resulting in a net decrease in the number of free radicals. Typically two free radicals combine to form a more stable species, for example: $\mathrm{Cl} \cdot+\mathrm{Cl} \cdot \rightarrow 2 \mathrm{Cl}^{18}$

The formation of radicals may involve breaking of covalent bonds homolytically, a process that requires significant amounts of energy. For example, splitting $\mathrm{H} 2$ into $2 \mathrm{H}^{\cdot}$ has a $\Delta \mathrm{H}^{\circ}$ of $+435 \mathrm{~kJ} / \mathrm{mol}$, and $\mathrm{Cl} 2$ into $2 \mathrm{Cl}$. has a $\Delta \mathrm{H}^{\circ}$ of $+243 \mathrm{~kJ} / \mathrm{mol} .{ }^{19}$ This is known as the homolytic bond dissociation energy, and is usually abbreviated as the symbol $\mathrm{DH}^{\circ}$. The bond energy between two covalently bonded atoms is affected by the structure of the molecule. Homolytic bond cleavage most often happens between two atoms of similar electronegativity. However, propagation is a very exothermic reaction. Radicals may also be formed by single electron oxidation or reduction of an atom or molecule. An example is the production of superoxide by the electron transport chain..$^{20}$

\section{Free radical-targets}

Free radicals attack three main cellular components.

\section{Lipids}

Peroxidation of lipids in cell membranes can damage cell membranes by disrupting fluidity and permeability. Lipid peroxidation can also adversely affect the function of membrane bound proteins such as enzymes and receptors.

\section{Proteins}

Direct damage to proteins can be caused by free radicals. This can affect many kinds of protein, interfering with enzyme activity and the function of structural proteins.

\section{DNA}

Fragmentation of DNA caused by free radical attack causes activation of the poly (ADP-ribose) synthetase enzyme. This splits Nicotinamide-adenine dinucleotide $\left(\mathrm{NAD}^{+}\right)$to aid the repair of DNA. 
However, if the damage is extensive, $\mathrm{NAD}^{+}$levels may become depleted to the extent that the cell may no longer be able to function and dies. The site of tissue damage by free radicals is dependent on the tissue and the reactive species involved. Extensive damage can lead to death of the cell; this may be by necrosis or apoptosis depending on the type of cellular damage. When a cell membrane or an organelle membrane is damaged by free radicals, it loses its protective properties. This puts the health of the entire cell at risk. ${ }^{21}$

\section{Damaging effects}

Cells normally defend themselves against ROS damage through the use of enzymes such as superoxide dismutase and catalase. Small molecule antioxidants such as ascorbic acid (vitamin C), uric acid, and glutathione also play important roles as cellular antioxidants. Similarly, polyphenol antioxidants assist in preventing ROS damage by scavenging free radicals. The negative effects of ROS on cell metabolism include roles in programmed cell death and apoptosis, whereas positive effects include induction of host defense genes and mobilization of ion transport systems. In particular, platelets involved in wound repair and blood homeostasis release ROS to recruit additional platelets to sites of injury. These also provide a link to the adaptive immune system via the recruitment of leukocytes. Reactive oxygen species are involved in cardiovascular disease, hearing impairment via cochlear damage induced by elevated sound levels, ototoxicity of drugs such as cisplatin, and in congenital deafness in both animals and humans. ${ }^{22}$ Generally, harmful effects of reactive oxygen species on the cell are most often:

a. Damage of DNA

b. Oxidations of polydesaturated fatty acids in lipids

c. Oxidations of amino acids in proteins

d. Oxidatively inactivate specific enzymes by oxidation of cofactors

\section{Reactive oxygen species (ROS)}

Reactive oxygen species (ROS) are very small molecules and are highly reactive due to the presence of unpaired valence shell electrons. ROS is formed as a natural byproduct of the normal metabolism of oxygen and have important roles in cell signaling. However, during times of environmental stress ROS levels can increase dramatically, which can result in significant damage to cell structures. Platelets involved in wound repair and blood homeostasis release ROS to recruit additional platelets to sites of injury. Generally, harmful effects of reactive oxygen species on the cell are most often like-Damage of DNA, oxidations of polydesaturated fatty acids in lipids, oxidations of amino acids in proteins, oxidatively inactivates specific enzymes by oxidation of co-factors(Table 1). ${ }^{23}$

Table I Reactive oxygen species and their corresponding neutralising antioxidants and also additional antioxidants

\begin{tabular}{llll}
\hline $\begin{array}{l}\text { Reactive } \\
\text { oxygen species } \\
\text { (ROS) }\end{array}$ & \multicolumn{2}{c}{ Antioxidants (endogenous) } & \multicolumn{2}{c}{$\begin{array}{l}\text { Antioxidants } \\
\text { (exogenous) }\end{array}$} \\
\cline { 2 - 3 } & Direct role & Indirect role & \\
& & $\begin{array}{l}\text { Transferin } \\
\text { (iron) Vitamin }\end{array}$ & Vitamin C, \\
Hydrogen & $\begin{array}{l}\text { Catalase } \\
\text { (cofactor) }\end{array}$ & $\begin{array}{l}\text { Ferritin (iron) } \\
\text { beta-carotene, } \\
\text { Myoglobin (iron }\end{array}$ & lipoic acid \\
\hline
\end{tabular}

\begin{tabular}{|c|c|c|c|}
\hline $\begin{array}{l}\text { Reactive } \\
\text { oxygen species } \\
\text { (ROS) }\end{array}$ & \multicolumn{2}{|c|}{ Antioxidants (endogenous) } & \multirow{2}{*}{ 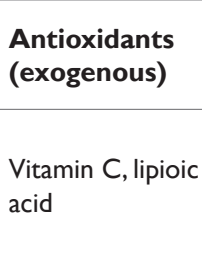 } \\
\hline Hydroxyl radical & $\begin{array}{l}\text { Glutathione } \\
\text { peroxidase } \\
\text { (cofactor } \\
\text { selenium) }\end{array}$ & & \\
\hline Lipid peroxidase & $\begin{array}{l}\text { Glutathione } \\
\text { peroxidase } \\
\text { (cofactor } \\
\text { selenium) }\end{array}$ & & $\begin{array}{l}\text { Vitamin E, beta } \\
\text { carotene }\end{array}$ \\
\hline $\begin{array}{l}\text { Superoxide } \\
\text { radical }\end{array}$ & $\begin{array}{l}\text { Superoxidase } \\
\text { dismutase } \\
\text { (Cofactor } \\
\mathrm{Cu} / \mathrm{Zn} / \mathrm{Mn} \text { ) }\end{array}$ & $\begin{array}{l}\text { Ceruloplasmin } \\
\text { (Metallothionin } \\
\text { Albumin }\end{array}$ & Vitamin C \\
\hline $\begin{array}{l}\text { Pro-oxidant/ } \\
\text { Antioxidants } \\
\text { equilibrium }\end{array}$ & $\begin{array}{l}\text { Thiols (GSH, } \\
\text { Lipoic acid, } \\
\text { N-acetyl } \\
\text { cysteine) } \\
\text { NADH and } \\
\text { NADPH } \\
\text { Ubiquinone }\end{array}$ & $\begin{array}{l}\text { Bilirubin uric } \\
\text { acid }\end{array}$ & Flavonoids \\
\hline
\end{tabular}

These are the list of antioxidants that help to include in the diet and manage to scavenge the free radical that can develop the steps of neurodegeneration. ${ }^{39}$

\section{Free radicals in beneficial role}

a. Free radicals perform many critical functions in our bodies in controlling the flow of blood through our arteries, to fight infection, to keep our brain alert and in focus.

b. Phagocytic cells involved in body defense produce and mobilize oxygen free radicals to destroy the bacteria and other cells of foreign matter which they ingest.

c. Similar to antioxidants, some free radicals at low levels are signaling molecules, i.e. they are responsible for turning on and off of genes.

d. Some free radicals such as nitric oxide and superoxide are produced in very high amount by immune cells to poison viruses and bacteria.

e. Some free radicals kill cancer cells. In fact certain cancer drugs aim in increasing the free radical amount in body.

\section{Defensive systems against free radicals}

All aerobic forms of life maintain elaborate anti-free-radical defense systems, also known as antioxidant systems. Enzymes: The defense enzyme, superoxide dismutase (SOD), takes hold of molecules of superoxide-a particularly destructive free radical-and changes them to a much less reactive form. SOD and another important antioxidant enzyme set, the glutathione system, work within the cell. Circulating biochemical's like uric acid and ceruloplasmin react with free radicals in the intercellular spaces and bloodstream.

Self repair: The body also has systems to repair or replace damaged building blocks of cells. Most protein constituents in the cell are completely replaced every few days. Scavenger enzymes break used and damaged proteins into their component parts for reuse by the cell.

Nutrients: Vitamins and other nutrients neutralize the oxy radicals' and serves as second line of defense. Among the many substances used are Vitamins $\mathrm{C}$ and E, beta-carotene, and bioflavonoids. ${ }^{24}$ 


\section{Antioxidants}

Free radical or oxidative injury may be a fundamental mechanism underlying a number of human neurologic diseases. ${ }^{25}$ Oxidative stress (OS) has been implicated in the pathophysiology of many neurological, particularly neurodegenerative diseases. OS can cause cellular damage and subsequent cell death because the reactive oxygen species (ROS) oxidize vital cellular components such as lipids, proteins, and DNA. Therapy using free radical scavengers (antioxidants) has the potential to prevent, delay, or ameliorate many neurologic disorders. However, the biochemistry of oxidative pathobiology is complex, and optimum antioxidant therapeutic options may vary and need to be tailored to individual diseases. In vitro and animal model studies support the potential beneficial role of various antioxidant compounds in neurologic disease. However, the results of clinical trials using various antioxidants, including vitamin $\mathrm{E}$, tirilazad, $\mathrm{N}$-acetylcysteine, and ebselen, have been mixed. Moreover, therapy with antioxidants may need to be given early in chronic insidious neurologic disorders to achieve an appreciable clinical benefit. Pre-disease screening and intervention in at-risk individuals may also need to be considered in the near future.

\section{Oxidative stress}

Oxidative stress is a general term used to describe a serious imbalance between the production of reactive oxygen species (ROS) and reactive nitrogen species (RNS) on the one hand, and the levels of antioxidant defences on the other. Any prolonged imbalance results in oxidative damage to cells, tissues, and organs. ${ }^{26}$ Intracellular ROS are produced in mitochondria as a by-product of normal cellular metabolism. ${ }^{23}$ The cytoplasm via the xanthine oxidase and reduced nicotinamide adenosine dinucleotide phosphate (NADPH) oxidase (NOX) pathways. ${ }^{27,28}$ In addition, oxidative stress may result from exposure to a variety of agents present in the environment. External sources of ROS include radiation, UV light, chemical reagents, pollution, cigarette smoke, drugs of abuse, and alcohol. ${ }^{29}$

At low or moderate levels, ROS and RNS exert beneficial effects on cellular responses and immune function. However, at high concentrations, they cause oxidative stress, and subsequent damage to proteins, lipids, and DNA. ${ }^{30}$ The brain is particularly vulnerable to oxidative damage because of its high oxygen utilisation, its high content of oxidisable polyunsaturated fatty acids, and the presence of redox-active metals (such and copper and iron) ) $^{31,32}$ It is well established that oxidative stress tends to increase with age as the antioxidant defense system becomes progressively less efficient in handling the production of ROS and RNS. It has been proposed that this age induced increase in oxidative stress may contribute and/or accelerate neurodegeneration in several neurodegenerative diseases, including Huntington disease. ${ }^{33}$

\section{ROS}

Why the need of the free radical in neurodegeneration: The harmful effects of ROS cause damage to macromolecules such as proteins, lipids, polysaccharides or nucleic acids, are termed oxidative stress. The intrinsic properties of neurons make them highly vulnerable to the detrimental effects of ROS: high metabolic rates; a rich composition of fatty acids prone to peroxidation; high intracellular concentrations of transition metals, capable of catalyzing the formation of reactive hydroxyl radicals; low levels of antioxidants; and reduced capability to regenerate. Neurons have intense energy demands which are met by mitochondria. Mitochondria are both targets and important sources of ROS. It has been shown that oxidative stress stimulates mitochondrial fission; the addition of hydrogen peroxide to cultured cerebellar granule neurons induced mitochondrial fragmentation within one hour of treatment. ${ }^{34}$

It was also shown that nitric oxide causes increased mitochondrial fission in neurons, prior to the onset of neuronal loss in a mouse model of stroke. On the other hand, expression of Mfn or a dominant negative Drp1 in cultured neurons, was protective against oxidative insults. ${ }^{35}$

The generation of ROS appears to be increased in damaged mitochondria, and in cells with compromised mitochondrial function. Acute exposure to relatively high levels of ROS, especially in the presence of calcium, can induce the mitochondrial permeability transition, uncouple oxidative phosphorylation with catastrophic effects on mitochondrial energetics, and contribute to cytotoxicity via necrosis and/or apoptosis. Oxidative stress within mitochondria can lead to a vicious cycle in which ROS production progressively increases leading, in turn, to progressive augmentation of damage. Nucleic acid oxidation occurs in neurons during disease and is detected as elevated levels of 8-hydroxy-2-deoxyguanosine (8OHDG) in DNA and 8-hydroxyguanosine in RNA. Hydroxyl radicalmediated DNA damage often results in strand breaks, DNA-protein crosslinking, and base-modifications. All of these events can lead to neuronal injury. It is known that generation of ROS results in an attack not only on DNA, but also on other cellular components involving polyunsaturated fatty acid residues of phospholipids, which are extremely sensitive to oxidation. ${ }^{36}$

Once formed, peroxyl radicals (ROO-) can be rearranged via a cyclisation reaction to endoperoxides (precursors of malondialdehyde) with the final product of the peroxidation process being malondialdehyde (MDA). The major aldehyde product of lipid peroxidation other than malondialdehyde is 4-hydroxy-2-nonenal (HNE). Increased production of ROS also results in protein oxidation. The side chains of all amino acid residues of proteins, in particular cysteine and methionine residues of proteins are susceptible to oxidation by the action of ROS/reactive nitrogen species (RNS). ${ }^{37}$

Oxidation of cysteine residues may lead to the reversible formation of mixed disulfides between protein thiol groups (-SH) and low molecular weight thiols, in particular glutathione (GSH, S-glutathiolation). The concentration of carbonyl groups, generated by many different mechanisms is a good measure of ROS-mediated protein oxidation. The generation of isoprostanes has been shown to be a sensitive measure of lipid peroxidation, which are increased in cerebrospinal fluid (CSF) of HD patients..$^{38}$ Oxidative stress increases with age in the brain and the ability of cells to respond to oxidative protein damage also declines, contributing to the buildup of oxidatively damaged proteins. ROS are often present in brain regions affected by neurodegenerative diseases. Increased oxidative alterations to proteins such as $\alpha$-synuclein in Parkinson's disease, $\beta$-amyloid in Alzheimer's disease and SOD1 in Amylotrophic Lateral Sclerosis may result in increased protein misfolding and impaired degradation, leading to toxic accumulation of insoluble aggregates in the diseased brains and an exacerbation of neurodegeneration. ${ }^{39}$

\section{Oxidative damage in Huntington and its evidence}

Studies in both HD patients and experimental models of HD support a role for oxidative stress and ensuing mitochondrial dysfunction in mediating the neuronal degeneration observed in HD. Oxidative damage in HD has been previously reviewed in detail. ${ }^{40,41}$ 


\section{Antioxidant therapies used in HD}

The accumulation of ROS in neurons, and subsequent oxidative stress are attenuated by free radical scavengers, which can be categorized as enzymatic or non-enzymatic antioxidants. Enzymatic antioxidants constitute one of the defense mechanisms against free radicals. These include superoxide dismutase (SOD), glutathione peroxidase $(\mathrm{Gpx})$ and catalase (CAT). Non-enzymatic antioxidants are represented by ascorbic acid (Vitamin C), $\alpha$-tocopherol (Vitamin E), glutathione (GSH), retinoic acid, carotenoids, flavonoids, and other antioxidants. The therapeutic approaches tested in vitro or in toxin or transgenic models of HD are given below-

\section{In vitro studies}

a. Metalloporphyrins, metal-containing catalytic antioxidants, have emerged as a novel class of potential therapeutic agents that scavenge a wide range of reactive oxygen species. A manganese porphyrin has been reported to significantly reduce cell death in an in vitro chemical model of HD. ${ }^{42}$

b. Ascorbic acid (Vitamin C) is a potent antioxidant obtained exogenously, which oxidizes readily to dehydroxyascorbic acid in the presence of reactive oxygen species. Treating cultured rodent cortical neurons with glutamate resulted in significant neurodegeneration, which was completely rescued with ascorbic acid co-treatment. ${ }^{43}$

c. $\alpha$-tocopherol (Vitamin E) is also a potent antioxidant. Using a neuronal cell-based assay, glutamate-induced neuronal death was significantly attenuated in a dose-dependent manner by $\alpha$-tocopherol. Also, treatment with idebenone in this in vitro model resulted in complete neuroprotection in a dose-dependent manner. ${ }^{44}$

d. Melatonin, which is an excellent scavenger of hydroxyl, carbonate, reactive nitrogen species, and other organic radicals, has demonstrated significant neuroprotection in the kainic acid rodent model of neurodegeneration. Melatonin significantly reduced DNA damage and improved neuronal survival. In another study using the 3-NP model of HD, melatonin treatment significantly ameliorated the increase in lipid peroxidation, protein carbonyls and SOD activity within the striatum. ${ }^{45}$

e. Selenium, is an essential element required by glutathione peroxidase to form the active enzyme. Selenium dose-dependently reduced lipid peroxidation and significantly improved neuronal morphology within the striatum of rats treated with quinolinic acid, an N-methyl-D-aspartate antagonist that results in striatal neurodegeneration. ${ }^{46}$

f. Creatine is a naturally occurring compound that has been shown to act as an antioxidant. Creatine also buffers intracellular energy reserves through its intermediate, phosphocreatine (PCr); stabilizes intracellular calcium; and inhibits activation of the mitochondrial transition pore. Creatine supplementation significantly reduces striatal lesion volumes produced by the neurotoxins 3-NP and malonate. The antioxidant compound CoQ10 also demonstrated efficacy in murine models of HD. CoQ10, ubiquinone, is a lipidsoluble benzoquinone which, when reduced to ubiquinol, which possesses significant antioxidant potential. In addition, CoQ10 can induce increases in vitamin $\mathrm{E}$, enhancing its antioxidant capacity. Using the mitochondrial toxins malonate. g. FK-506 (also known as Tacrolimus or Fujimycin) is an immunosuppressive drug mainly used to lower allograft rejection and also in topical preparations. Recently, the neuroprotective effects of FK-506 were reported in 3-NP model of HD. FK-506 treatment significantly reduced behavioral deficits, MDA levels, nitrite concentration, and restored antioxidant enzyme levels of SOD and catalase, and levels of dopamine and norepinephrine in the striatum, cortex, and hippocampus.

h. Lycopene, a carotenoid pigment and phytochemical naturally found in fruits and vegetables, reduced oxidative stress markers and improved behavior in a 3-NP induced rodent model of HD.

\section{In transgenic mouse models of HD}

a. Lipoic acid is an essential cofactor for many enzyme complexes and is present in mitochondria as the cofactor for pyruvate dehydrogenase and alpha-ketoglutarate dehydrogenase. It is an effective antioxidant and has been used to treat disease associated with impaired energy metabolism. It also induces the Nrf-2/ ARE pathway. In both the R6/2 and N171-82Q transgenic mouse models of $\mathrm{HD}$, dietary supplementation with lipoic acid resulted in significant extension of survival, and delayed weight loss in N171$82 \mathrm{Q}$ transgenic mice. Pyruvate plays a major role in glycolysis, and also possesses significant antioxidant capacity. Treatment with dichloroacetate, which stimulates pyruvate dehydrogenase, improved the HD phenotype of both R6/2 and N171-82Q mice, suggesting that, in addition to its antioxidant capacity, pyruvate may promote neuroprotection by improving energetic potential. ${ }^{47}$

b. BN82451 is a brain-permeable compound that exerts neuroprotective effects, including the inhibition of lipid peroxidation. Administration of BN82451 in R6/2 mice resulted in a significant extension of survival, significant improvements in motor function, with improvements in gross morphology, striatal volume, and striatal neuronal areas, and a significant decrease in the number of ubiquitin positive aggregates in R6/2 mice as compared with untreated control mice.

c. Creatine exists in the cell both as free creatine and phosphocreatine (PCr) which together comprise the total creatine pool. In tissues with high energy requirements such as skeletal muscle and brain, $\mathrm{PCr}$ serves as a short term energy buffer in which adenosine diphosphate is phosphorylated to adenosine triphosphate. This phosphorogroup transfer is catalyzed by the important creatine kinase (CK) enzyme. Creatine supplementation in the R6/2 and the N171-82Q transgenic mouse models produced an improvement in motor performance, extended survival, attenuated the loss of body weight and brain weight and reduced neuronal atrophy.

d. L-carnitine (4-N-trimethylammonium-3-hydroxy-butyric acid) plays a role in the control of the mitochondrial acyl-coenzyme A/ coenzyme A ratio, peroxisomal oxidation of fatty acids, and also acts as a free-radical scavenger. It is effective in preventing cell membrane damage caused by reactive oxygen species. Recently, administration of a relatively high dose of L-carnitine to N171$82 \mathrm{Q}$ transgenic mice was shown to extend the survival, ameliorate motor performance, and decrease the number of intranuclear aggregates.

e. Grape seed phenolic extract (GSPE) treatment in both fly and R6/2 mouse models of HD effectively extended lifespan in both models, and reduced motor deficits in the R6/2 mice, demonstrating preclinical efficacy of GSPE in HD treatment. 
f. Synthetic triterpenoids, which are analogues of 2-Cyano-3,12Dioxooleana-1,9-Dien-28-Oic acid (CDDO), are of great interest because of their antioxidant and anti-inflammatory properties We recently showed that administrations of triterpenoids, which activate the Nrf2/ARE transcriptional pathways, are neuroprotective in the N171-82Q transgenic mouse model of HD. Triterpenoids significantly preserved the striatal volumes of the N171-82Q mice, by preventing the atrophy of the medium spiny neurons, and they rescued behavioral deficits, extended survival, attenuated peripheral pathology, and reduced $8-\mathrm{OHdG}, \mathrm{MDA}$ and 3 -nitrotyrosine immune-reactivity in the striatum. ${ }^{48,49}$

\section{Conclusion}

As we know that neurodegenerative disorders is an important source of morbidity and mortality in human mankind. Free radical involvement offers a novel therapeutic target in the management of Huntington diseases. However, it will happen only if the mechanism of action of the free radical can be understood properly. The antioxidant therapy can be used over the free radical generation and target the free radical that are responsible for the neurodegeneration such as HD. Free-radical-mediated oxidative injury plays a major role in the defection of various diseases and trauma; chronic neurogenic and other neurodegeneration disease can be recognized.

We know that oxygen is an essential molecule for survival of majority of living organisms in the world as well as the inside the body. Oxidative stress mostly a harmful condition that occurs when there is an excess of free radicals will be generated or a decrease in antioxidant levels in the body. So, this review suggests the use of antioxidants to manage the disease state. Antioxidants quench oxidative stress by 1working to off-pair free radical generation and stopping them from starting the chain reactions that contribute to develop Huntington disease.

Oxidative stress (OS) has been responsible in the pathophysiology of various life threatening starts disease such as neurological and neurodegenerative diseases. Oxygen Species generally a free radical species can cause cellular damage and subsequent cell death because the reactive oxygen species which oxidize sensitive cellular components that makes a major part of proteins, lipids, ribose sugar and genetic material. However, the excitatory amino acid glutamate is the major amino acid that can prevent such kind of neurodegeneration that are responsible for Huntington disease populations that are at high risk, such as elderly and newly diagnosed patients. In this review we have to find the new way and the use of certain antioxidants to remove the wastes of free radical generation and scavenging properties of the antioxidants to manage in the disease and cure them.

\section{Acknowledgements}

None.

\section{Conflict of interest}

The author declares that there is no conflict of interest.

\section{References}

1. The Huntington's Disease Collaborative Research Group. A nove gene containing a rinucleotide repeat that is expanded and unstable on Huntington's disease chromosomes. Cell. 1993;72:971-983.

2. Ross CA, Tabrizi SJ. Huntington's disease: from molecular pathogenesis to clinical treatment. Lancet Neurol. 2011;10(1):83-98.
3. Roze E, Bonnet C, Betuing S, et al. Huntington's disease. Adv Exp Med Biol. 2010;685:45-63.

4. Rosas HD, Salat DH, Lee SY, et al. Complexity and heterogeneity: what drives the ever-changing brain in Huntington's disease? Ann NY Acad Sci. 2008;1147:196-205

5. Cowan CM, Raymond LA. Selective neuronal degeneration in Huntington's disease. Curr Top Dev Biol. 2006;75:25-71.

6. Walker FO. Huntington's Disease. Semin Neurol. 2007;27(2):143-150.

7. Berardelli A, Noth J, Thompson PD, et al. Pathophysiology of chorea and bradykinesia in Huntington's disease. Mov Disord. 1999;14(3):398-403.

8. $\mathrm{Li} \mathrm{SH}, \mathrm{Li} \mathrm{XJ}$. Huntingtin and its role in neuronal degeneration. Neuroscientist. 2004;10:467-475.

9. Factor SA, Friedman JH. The emerging role of clozapine in the treatment of movement disorders. Mov Disord. 1997;12:483-496.

10. Kieburtz K. Antiglutamate therapies in Huntington's disease. J Neural Transm Suppl. 1999;55:97-102.

11. Butler R, Bates GP. Histone deacetylase inhibitors as therapeutics for polyglutamine disorders. Nat Rev Neurosci. 2006;7:784-796.

12. Bonelli RM, Wenning GK. Pharmacological management of Huntington's disease: an evidence-based review. Curr Pharm Des. 2006;12(21):27012720 .

13. Stadtman ER. Role of oxidant species in aging. Curr Med Chem. 2004;11(9):1105-1112.

14. Lastres-Becker I, de Miguel R, De Petrocellis L, et al. Compounds acting at the endocannabinoid and/or endovanilloid systems reduce hyperkinesias in a rat model of Huntington's disease. J Neurochem. 2003;84(5):10971109 .

15. Fernandez-Ruiz J. The endocannabinoid system as a target for the treatment of motor dysfunction. Br J Pharmacol. 2009;156:1029-1040.

16. Sherki Y.G, Melamed E, Offen D. Oxidative stress inducedneurodegenerative diseases: the need for antioxidants that penetrate the blood brain barrier. Neuropharmacology. 2001;40(8):959-975.

17. P Pacher, JS Beckman, L Liaudet. Nitric oxide and peroxynitrite in health and disease. Physiol Rev.1997;87(1):315-424.

18. Parth's Pathophysiology: Concepts of Altered Health States. 7th edition. USA: Lippincott Williams \& Wilkins; 2008: 645.

19. S Laganiere, BP Yu. Modulation of membrane phospholipid fatty acid composition by age and food restriction. Gerontology. 1993;39(1):7-18.

20. D. Harman. A biologic clock: the mitochondria? J Am Geriatrics Society 1972;20(4):145-147.

21. $P$ Larsen. Aging and resistance to oxidative damage in Caenorhabditis elegans. Proc Natl Acad Sci U S A. 1993;90(19):8905-8909.

22. LA MacMillan-Crow, JA Thompson. Peroxynitrite--mediated inactivation of manganese uperoxide dismutase involves nitration and oxidation of critical tyrosine residues. Biochemistry. 1998;37:1613-1622.

23. Toxicology of the Human Environment - the critical role of free radicals. Taylor and Francis: London; 2000.

24. F Krotz, HY Sohn, T Gloe, et al. Oxidase-dependent platelet superoxide anion release increases platelet recruitment. Blood. 2002;100(3):917-924.

25. Abheri Das Sarma,m Buring JE, Peto R. Antioxidant Vitamins Benefits Not Yet Proved (editorial). International Journal of pharma Sciences and Research. 2010;1(3):185-192.

26. Delanty N. Antioxidant Therapy in Neurologic Disease. Arch Neurol. 2000;57:1265-1270 
27. Sies H. Oxidative stress: from basic research to clinical application. Am J Med. 1991;91:31S-38S.

28. Halliwell B, Gutteridge JM. Role of free radicals and catalytic metal ions in human disease: an overview. Methods Enzymol. 1990;186:1-85.

29. Gao HM, Liu B, Zhang W, et al. Critical role of microglial NADPH oxidase-derived free radicals in the in vitro MPTP model of Parkinson's disease. FASEB J. 2003;17:1954-1956.

30. Infanger DW, Sharma RV, Davisson RL. NADPH oxidases of the brain: distribution, regulation, and function. Antioxid Redox Signal. 2006;8:1583-1596.

31. Zadak Z, Hyspler R, Ticha A. Antioxidants and vitamins in clinical conditions. Physiol Res. 2009;58:S13-S17.

32. Halliwell B. Biochemistry of oxidative stress. Biochem Soc Trans. 2007;35:1147-1150.

33. Gerlach M, Ben-Shachar D, Riederer P, et al. Altered brain metabolism of iron as a cause of neurodegenerative diseases? J Neurochem. 1994;63(3):793-807.

34. Halliwell B. Reactive oxygen species and the central nervous system. $J$ Neurochem. 1992;59(5):1609-1623.

35. Sokoloff L. Energetics of functional activation in neural tissues. Neurochem Res. 1999;24(2):321-329.

36. Valko M, Leibfritz D, Moncol J, et al. Free radicals and antioxidants in normal physiological functions and human disease. Int J Biochem Cell Biol. 2007;39(1):44-84

37. Jahani-Asl A, Cheung EC, Neuspiel M, et al. Mitofusin 2 protects cerebellar granule neurons against injury-induced cell death. $J$ Biol Chem. 2007;282(33):23788-23798.

38. Barsoum MJ, Yuan H, Gerencser AA, et al. Nitric oxide-induced mitochondrial fission is regulated by dynamin-related GT Pases in neurons. EMBO J. 2006;25(16):3900-3911.
39. Siems WG, Grune T, Esterbauer H. 4-Hydroxynonenal formation during ischemia and reperfusion of rat small intestine. Life Sci. 1995;57(8):785789.

40. Montine TJ, Beal MF, Robertson D, et al. Cerebrospinal fluid F2isoprostanes are elevated in Huntington's disease. Neurology. 1999;52(5):1104-1105.

41. Singh R.P, Sharad S, Kapur S. Free Radicals and Oxidative Stress in neurodegenerative diseases: relevance of dietary antioxidants. Indian Academy of Clinical Medicine. 2004;5:218-225.

42. Browne SE, Ferrante RJ, Beal MF. Oxidative stress in Huntington's disease. Brain Pathol. 1999;9(1):147-163.

43. Beal MF, Ferrante RJ. Experimental therapeutics in transgenic mouse models of Huntington's disease. Nat Rev Neurosci. 2004;5(5):373-384.

44. Stack EC, Matson WR, Ferrante RJ. Evidence of oxidant damage in Huntington's disease: translational strategies using antioxidants. Ann NY Acad Sci. 2008;1147:79-92.

45. Albin RL, Reiner A, Anderson KD, et al. Preferential loss of striatoexternal pallidal projection neurons in presymptomatic Huntington's disease. Ann Neurol. 1992;31(4):425-443.

46. Ferrante RJ, Kowall NW, Beal MF, et al. Selective sparing of a class of striatal neurons in Huntington's disease. Science. 1985;230(4725):561563.

47. Ferrante RJ, Beal MF, Kowall NW, et al. Sparing of acetylcholinesterasecontaining striatal neurons in Huntington's disease. Brain Res. 1987;411(1):162-166.

48. White JK, Auerbach W, Duyao MP, et al. Huntingtin is required for neurogenesis and is not impaired by the Huntington's disease CAG expansion. Nat Genet. 1997;17(4):404-410.

49. Johri A, Beal MF. Antioxidants in Huntington's disease. Biochim Biophys Acta. 2012;1822(5):664-674. 